However, it is worrisome to note that the proposed bill falls short in some vital areas. It failed to provide a clear statement on the promotion of fundamental rights of people who are mentally ill and does not specifically guarantee the rights of users of mental health services in relation to issues like confidentiality. It is silent on provisions regarding 'non-protesting' patients and involuntary treatment in community settings. The proposed law does not regulate special treatments such as electroconvulsive therapy (ECT), the use of seclusion and restraint, issues related to clinical and experimental research (consent in particular), and socio-political issues such as discrimination, housing, employment, social security, civil issues (e.g. voting rights, parental rights) as well as protection of other vulnerable groups, like women and ethnic minorities. When all these areas are further distilled into component parts, the overall level of compliance with WHO recommendations may be far lower than is superficially suggested by this overview.

In spite of the foregoing, the prospects for a successful revision of the existing law are brightened by a variety of local factors. Currently, the Association of Psychiatrists in Nigeria (APN), a major stakeholder in mental healthcare, is at the vanguard of the mental health law reform and mental health advocacy is gaining momentum. In recent times, a desk officer for mental health had been appointed at the Federal Ministry of Health, partly with a mandate to work with stakeholders towards collating inputs for the bill to be sponsored as an executive bill. Furthermore, the National Human Rights Commission subscribes to existing charters that strengthen human rights and thus constitutes a potential ally and stakeholder in the current effort to revise the existing legislation.

The WHO recommendations of developing country-specific mental health legislation that is needs-based, driven by human rights, collaborative in orientation and culturally sensitive offer practical guidelines for the construction of a new law. Coupled with these are the abundant legislation-related resources which the WHO has made widely available and which afford the opportunity to learn from more recent legislation in other countries. Furthermore, current epidemiological data with which to identify mental health needs are available (Gureje et al, 2006) and provide a basis for the needs-based approach of the WHO.

In addition, the recommended technical competence required to draft new legislation is obtainable in the country. In line with the WHO recommendation, major stakeholders (including the medical directors of existing federal psychiatric hospitals in the country) are currently brainstorming and engaging the Federal Ministry of Health with a view to accelerating the passage of the bill.

\section{Conclusion}

It is important to state that to achieve the desired target of the passage of the proposed legislation, advocacy driven by all stakeholders must be given serious attention. This certainly will bring pressure to bear on the government and ensure speedy enactment of a new law that will meet contemporary benchmarks, improve mental healthcare delivery and provide a better basis for later legislative revisions that must come with time.

\section{References}

Gureje, O., Lasebikan, V. O., Kola, L., et al (2006) Lifetime and 12 -month prevalence of mental disorders in the Nigerian Survey of Mental Health and Well-Being. British Journal of Psychiatry, 188, 465-471.

Laws of Nigeria (1948) Lunacy ordinance, Vol. IV, Cap. 121 Government Printer.

Laws of Ogun State of Nigeria (2006) Lunacy law. Ogun State Ministry of Justice, LexisNexis Butterworths (PTY) Ltd.

Morakinyo, V. O. (1977) The law and psychiatry in Africa. African Journal of Psychiatry, 3, 91-98.

Ogunlesi, A. O., Ogunwale, A., De Wet, P., et al (2012) Forensic psychiatry in Africa: prospects and challenges. African Journal of Psychiatry, 15, 3-7.

United Nations (1948) Universal Declaration of Human Rights. United Nations.

United Nations (1991) Principles for the Protection of Persons with Mental Illness and the Improvement of Mental Health Care. Office of the United Nations High Commissioner for Human Rights.

WHO (2003) The Mental Health Context. Mental Health Policy and Service Guidance Package. World Health Organization. Available at http://www.who.int/mental_health/policy/services (accessed 1 March 2012).

WHO (2005) WHO Resource Book on Mental Health, Human Rights and Legislation. World Health Organization. Available at http://www. who.int/mental_health/policy (accessed 1 March 2012).
MENTAL HEALTHLAW PROFILE

\title{
Mental health legislation in Egypt
}

\author{
Nasser Loza FRCPsych and Mohamed El Nawawi MSc
}

Behman Hospital, Helwan, Cairo, Egypt, email nloza@behman.com
This paper first briefly reviews the history of psychiatric services in Egypt. It then details the legislation in place during the last years of the Mubarak regime and goes on to set out recent developments, in particular the Code of Practice introduced for the Mental Health Act of 2009.

\section{Historical background}

The earliest reference to the care in specialised institutions of people with a mental illness dates back to Fatimid Egypt and the establishment of the Bimaristan in the 13th century, which still stands today in central Cairo. Throughout the Islamic 
era in Egyptian history, care of people with a mental illness appears to have been community based, while institutional care for those suffering from mental health problems was within general hospitals. The building of asylums away from residential areas, to separate those with mental illness from their communities, began only in the late 19th century. This followed a visit by Urquhart and Tuke from the Royal Medico-Psychological Association in 1879 (see Tuke \& Urquhart, 1879).

The beginning of the British protectorate in Egypt led to the modern practice of asylum care. The Department of Mental Health at the Medical School of Cairo University was closed in 1880 and psychiatrists were directed towards the newly built asylums, where training for their profession was more vocational than academic. It was at this point that regulations organising the involuntary admission of a person with a mental illness were needed. With only one asylum in Egypt, however, the regulations were internally set by the superintendent.

The first reference to a draft of a proposed lunacy law appears in the 1921 annual report from the lunacy division of the Egyptian Ministry of the Interior (Government Press Cairo, 1922). Dr John Warnock, superintendent at El Abbassia Hospital, appears to have modelled this draft on Britain's 1890 Lunacy Act. Asylums in Egypt were regulated by the Central Administration for Lunatics, which granted licences, organised visits and reviewed petitions from the families of detained lunatics.

The Egyptian parliament ratified the first Mental Health Act in 1944. This legislation addressed the involuntary detention of psychotic patients, second opinions, consent to treatment and appeals. The law formed the basis of hospital practice of psychiatry for about 30 years. Nonetheless, by the 1980 s psychiatric hospitals were detaining numerous patients who did not suffer from psychotic disorders (largely because of loopholes in the legislation), but rather had addictions or behavioural disorders; in fact, the involuntary detention of patients on moral grounds became common practice (Zaki, 2009).

\section{The recent past}

By 2006, Egypt had 8000 in-patient beds for people presenting with mental illness, all on locked wards, and many of these patients stayed for decades. The Mental Health Act was not applied; instead, almost all patients were claimed to be voluntarily admitted as, nationally, only four were detained under the Act.

In 2006, a group of mental health professionals at the Ministry of Health, with the support of the World Health Organization (WHO) and funding from donors, including the Foreign Ministry of Finland, drafted a new Mental Health Bill. This was ratified in parliament in May 2009.

The Mental Health Act of 2009 (Law 71, published in the Official Gazette, issue 20, 14 May 2009) brought basic conceptual changes to the care of people with a mental illness in Egyptian institutions. This, like its predecessor, focused on the rights of those with a mental illness, independent second opinions from psychiatrists and patients' right to consent to treatment. The real change in the environment of mental hospitals followed the policy of opening the gates to visitors, the press and international professional organisations, such as the Royal College of Psychiatrists, the Arab Board of Psychiatry, the Institute of Psychiatry in London and the World Federation for Mental Health, which all offered to support the work. In parallel to this, another important step was to campaign and raise awareness regarding the rights of people with a mental illness to live in the community. A media campaign was launched using television, billboards and educational material in schools.

Initially, this started with the Ministry of Health's effort and funding; then the work gradually took off on its own, with non-governmental organisations (NGOs), human rights organisations and consumer representatives taking the lead.

By May 2009, the law was applied to all mental institutions in Egypt, and a Code of Practice (2010 - ministerial decree number 128) was completed. Training workshops were conducted throughout Egypt. The application of the new Act appeared to be well accepted by most hospital-based professionals but not fully supported by psychiatrists, who had practised without the implementation of the 1944 Mental Health Act for decades. Indeed, a body of resistance built up among clinicians, who felt that the new Act, by empowering patients, represented a threat to clinicians' judgement. In a culture that is essentially patriarchal, in a society that lived under a dictatorship, it is not surprising that doctors would want to retain their powers.

\section{On the brink of change}

In January 2011, young Egyptian activists went out on to the streets to demonstrate against police brutality and eventually demanded a change of political system. The Mubarak regime was replaced by the Supreme Council of Armed Forces and parliamentary elections took place in November 2011 (with an overwhelming majority of religious parties). A number of liberal attitudes perceived as being Western ideologies came into question in the new Egypt, including laws addressing women's rights, the protection of children and rights pertaining to sexual orientation. With an increased level of street violence, accompanied by a prevailing mixed culture of military and religious ideologies, the current government is unlikely to uphold these values.

The Code of Practice of the Mental Health Act was redrafted in 2011 (ministerial decree number 210) by a working group, which chose to relax the requirements for patients' informed consent to treatment, from a written signed form to a written entry in the patient's notes stating that the patient has verbally consented. The new Code allows the compulsory use of psychotropic medication to facilitate bringing people to hospital from their 
private homes without prior permission from the district attorney. Involuntary electroconvulsive therapy (ECT) without second opinion for up to three initial sessions became legitimate. The role of patients' rights committees was diminished.

Violations of the human rights of people who are mentally ill are recognised globally (Drew et al, 2011). The Egyptian experience underlines the importance of public education and community participation in the drafting of new legislation. Ideally, laws are ratified to fulfil society's need for regulation. Bringing in new ideas of human rights and empowerment of service users becomes a challenge when the predominant culture does not fully acknowledge the rights of people with a mental illness.

Nevertheless, the process of drafting the Act with repeated conferences and workshops, lengthy parliamentary debates in the upper and lower houses, and substantial media coverage - has had an effect on the wider community's perception of people who are mentally ill, their place in society and their role. Patients are their own best advocates. Bringing a long-stay patient to meet with MPs in the Egyptian parliament was probably the loudest call that led to Egypt's Mental Health Act 2009.

\section{References}

Drew, N., Funk, M., Tang, S., et al (2011) Human rights violations of people with mental and psychosocial disabilities: an unresolved global crisis. Lancet, 378, 1664-1675.

Government Press Cairo (1922) Annual Report 1921, Lunacy Division, Egyptian Ministry of the Interior. Government Publications Office, Ministry of Finance.

Tuke, W. S. \& Urquhart, A. R. (1879) Two visits to the Cairo Asylum, 1877 and 1878. Journal of Mental Science, 25, 43-48.

Zaki, M. M. (2009) Egyptian legislation on the compulsory treatment of persons with psychiatric disorders: the old and the new. International Psychiatry, 6, 57-58.

\section{RESEARCH} PAPER

\title{
The 'revolving door': a profile of acute admissions at a South African psychiatric hospital
}

\author{
Zahir Vally ${ }^{1,2}$ and Nasera Cader ${ }^{1,2}$
}

'Lentegeur Psychiatric Hospital, Cape Town, South Africa, email z.vally@pgr.reading.ac.uk

${ }^{2}$ Department of Psychiatry and Mental Health, University of Cape Town, South Africa

The authors wish to thank Felicity Haigh for her assistance in completing the data collection.
This paper reports admission rates within the acute service at a major South African tertiary psychiatric facility, Lentegeur Psychiatric Hospital (LPH) in Cape Town.

The acute service in South African psychiatric hospitals is a challenging environment in which clinicians are faced with a multitude of factors that impinge on the efficacy of their interventions. This environment is characterised by lengthy and constantly growing waiting lists, an ever-present pressure to vacate hospital beds for incoming patients and a noticeable increase in the clinical presentation of severe Axis I diagnoses (Strebel $e t$ $a l, 1999)$. Moreover, the social context from which these patients emerge compounds the burden on services and should be considered in planning treatment. There is widespread poverty, the vast majority reside in informal housing on the outskirts of the urban cities, there is mass unemployment, some of the highest rates of substance misuse in the world, and an increasing prevalence of HIV and other communicable diseases. This is an important consideration, given that there is emerging evidence from low- and middle-income countries that mental ill-health is strongly associated with poverty and aspects of social deprivation
(Patel \& Kleinman, 2003). These external psychosocial factors in turn contribute to poor discharge planning, the premature discharge of patients who clinically would require a lengthier hospital stay and, ultimately, speedy readmission following discharge. This phenomenon is most often captured by the term 'revolving door', coined to describe patients who are admitted at least three times during their lifetime (Webb et al, 2007). Findings in the UK suggest that readmission rates among patients with severe psychiatric disorders are high: approximately $50 \%$ of patients admitted have had previous psychiatric admissions, and about $40 \%$ of patients require rehospitalisation within 1 year of discharge (Sweetman \& Davies, 2004).

There are a number of factors that appear to predict readmission. The majority of studies suggest that the number of previous psychiatric admissions is a good predictor of the risk of readmission (e.g. Webb et al, 2007; Bowersox, 2009). This is often the most reliable indicator. Of those readmitted, between one-half and two-thirds are readmitted within 12 months of discharge, suggesting that this is a high-risk period. The most critical point is 1 month after discharge. Higher rates of readmission are associated with a diagnosis of schizophrenia and other psychosis. A diagnosis of substance misuse or dependence is also associated 